\title{
Bilirubin: The toxic mechanisms of an antioxidant molecule
}

\author{
Constanza P. Soto Conti, M.D. ${ }^{a}$
}

\begin{abstract}
Hyperbilirubinemia is the most common reason for consultation and hospitalization in the neonatal period. It requires a timely initiation of an effective treatment because newborn infants are especially vulnerable to damage caused by bilirubin in the central nervous system due to the characteristics typical of this stage of life.

High bilirubin levels result in neurotoxicity and oxidative stress. However, molecular biology studies have demonstrated that bilirubin itself acts as a potent antioxidant.

The objective of this update is to review the processes whereby bilirubin causes cell damage and determine its beneficial antioxidant effects. Knowing these mechanisms may facilitate a more accurate indication of a customized, effective, and timely phototherapy. Until new scientific advances are made, phototherapy should be prescribed based on expert consensus.

Key words: bilirubin, neurotoxicity syndromes, antioxidants, oxidative stress, newborn.
\end{abstract}

http: / / dx.doi.org/ 10.5546/ aap.2021.eng.e18

To cite: Soto Conti CP. Bilirubin: The toxic mechanisms of an antioxidant molecule. Arch Argent Pediatr 2021; 119(1):e18-e25. a. Hospital Materno Infantil Ramón Sardá. Autonomous City of Buenos Aires, Argentina.

E-mail address:

Constanza P. Soto Conti, M.D.:

cleosc@yahoo.com

Funding:

None.

Conflict of interest:

None.

Received: 5-29-2020

Accepted: 8-18-2020

\section{GLOSSARY}

BVR: biliverdin reductase.

CNS: central nervous system.

DB: direct (or conjugated) bilirubin.

DNA: deoxyribonucleic acid.

FIB: free indirect bilirubin (or

bilirubin not bound to albumin).

FR: free radical.

$\mathrm{HO}$ : heme oxygenase.

IB: indirect (or unconjugated) bilirubin .

NBI: newborn infant.

PNBI: preterm newborn infant.

RNA: ribonucleic acid.

TNF- $\alpha$ : tumor necrosis factor alpha.

TSB: total serum bilirubin.

UGT1A1: uridine diphosphate

glucuronosyltransferase 1A1.

\section{INTRODUCTION}

Hyperbilirubinemia is the most common reason for consultation and hospitalization among newborn infants (NBIs), for whom it poses potential neurological consequences, sometimes severe and irreversible, especially if they are preterm NBIs (PNBIs). ${ }^{1}$ The cellular and molecular mechanisms whereby bilirubin causes central nervous system (CNS) damage have been described over the years. However, many questions remain to be answered. ${ }^{2-7}$

Bilirubin's beneficial antioxidant effects have been described with molecular biology techniques. In adults, an increase in total serum bilirubin (TSB) is a good prognosis factor in some conditions; it has even been speculated that it may be used in future therapeutic options..$^{8-11}$

It is worth noting that, in humans, bilirubin increases after birth, with the sudden changes in oxygen pressures and cellular oxidation. ${ }^{12}$ In turn, it is surprising that such potent antioxidant sometimes behaves in a toxic manner. 
NBIs should be closely monitored and receive a timely and effective treatment based on the best available resources: expert recommendations, at least until unanswered questions are resolved. ${ }^{13}$ An update on the damage-causing mechanisms and potential benefits of bilirubin may be useful for those who prescribe phototherapy on a daily basis to reflect about how bilirubin works in the setting of each NBI's characteristics.

\section{Bilirubin metabolism}

Bilirubin originates from heme-containing molecules. Eighty percent of heme comes from hemoglobin released by senescent red blood cells and an ineffective erythropoiesis. The remaining $20 \%$ comes from non-erythroid enzymatic sources: cytochromes, catalases, peroxidase, and tryptophan pyrrolase. ${ }^{14}$

In the cells of the reticuloendothelial system, the heme oxygenase (HO) enzyme hydrolyzes the heme molecule and turns it into a carbon monoxide molecule and a biliverdin molecule. Such reaction releases iron and consumes oxygen. Then, the biliverdin reductase (BVR) enzyme reduces biliverdin to bilirubin. Thus formed, bilirubin is known as unconjugated or indirect bilirubin (IB). This is a hydrophobic molecule, so it binds to albumin to be transported to the liver, where it is conjugated with glucuronic acid. The glucuronidation process turns it into conjugated or direct bilirubin (DB) and makes it watersoluble so it can be incorporated into bile, which carries it to the gut.

The gut bacterial flora turns DB into stercobilinogen and urobilinogen, pigments excreted through feces and urine. In the gut, before excretion, there is a possibility that DB may turn again into IB, due to the action of an enzyme called beta-glucuronidase, and then re-enter circulation as IB. This constitutes the enterohepatic circulation of bilirubin ${ }^{9,12}$ (Figure 1).

Bilirubin levels measured in blood account for

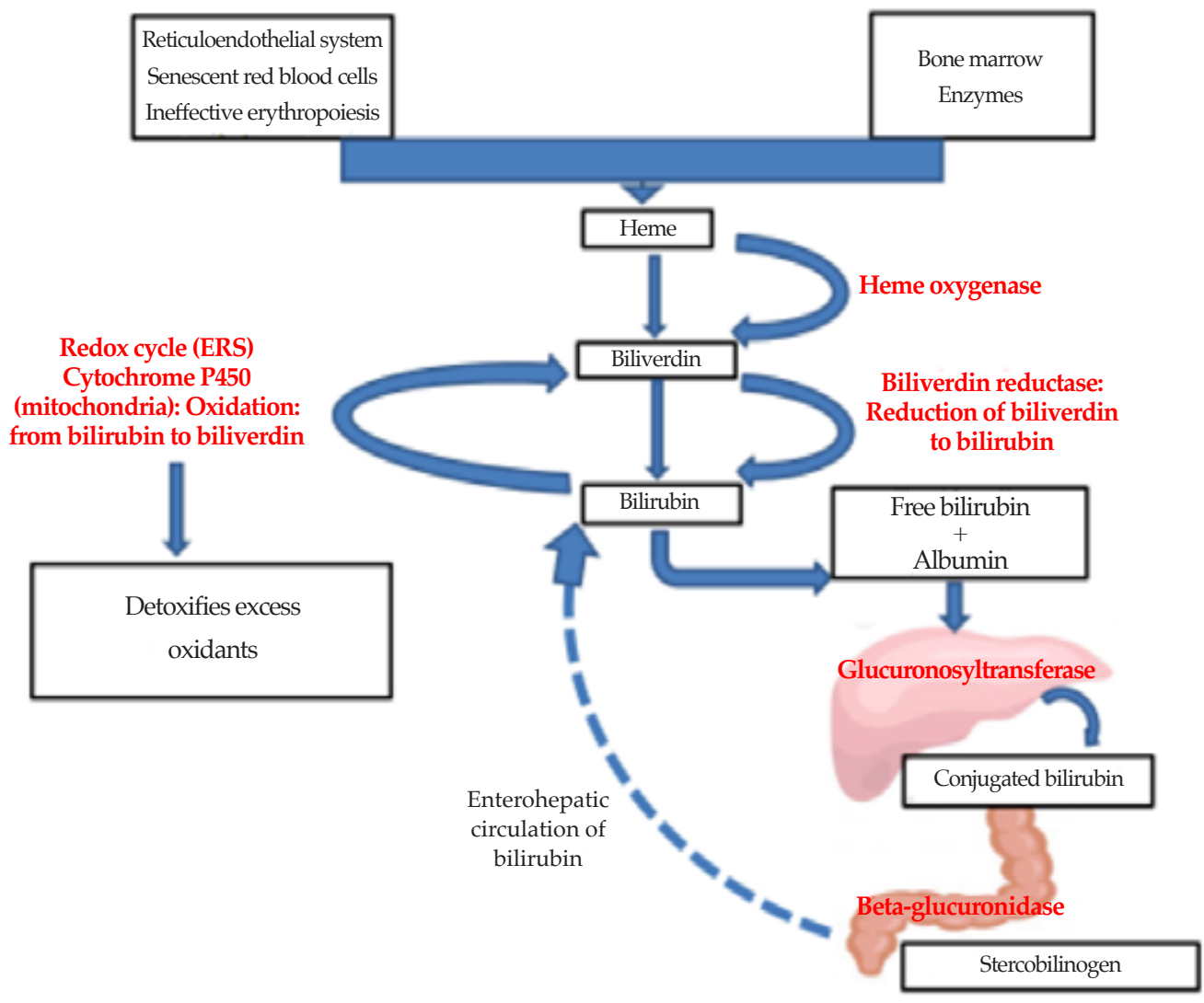

ERS: endoplasmic reticulum system.

The upper right part of the figure shows the level where the process occurs reversely with bilirubin being turned into biliverdin and capturing oxygen free radicals, thus resulting in an antioxidant effect. (Developed by the author). 
TSB concentrations, which is the sum of IB and DB. TSB levels include two fractions of IB: free indirect bilirubin (FIB), which accounts for $0.01 \%$ of $\mathrm{IB},{ }^{9}$ and albumin-bound bilirubin. In clinical practice, there is no easily accessible lab test to measure FIB, so therapeutic decisions are based on TSB levels. ${ }^{15}$

Bilirubin is lipid-soluble, passes through the membranes, and is incorporated to cells and organs. In the setting of intrauterine life, this characteristic allows bilirubin to cross the placental barrier from the fetus to the mother, who excretes it through the liver. ${ }^{14}$

\section{Vulnerability characteristics of newborn infants}

The human species is the only one that experiences a physiological bilirubin increase in the neonatal period. This is for two reasons: one is the physiological hemolysis caused by oxidative stress due to the change from placental to pulmonary oxygenation. In addition to this aspect, some individuals occasionally experience immune or hereditary hemolysis. The other reason is of metabolic origin, due to an increase in IB. The uridine diphosphate glucuronosyltransferase 1A1 (UGT1A1) enzyme conjugates bilirubin in the liver. There is evidence of the presence of this enzyme in the small intestine and of its role in the glucuronidation of bilirubin in the enterocyte.

Fujiwara et al. found, in exclusively breastfed NBIs, a suppression of UGT1A1 expression in the enterocyte, a lower glucuronidation, an increased enterohepatic circulation, and a greater IB availability. This mechanism, which has not been observed in formula-fed NBIs, highlights the physiological and antioxidant role of bilirubin in response to the oxidative stress caused by birth. ${ }^{14}$ In addition, some NBIs may have hematoma resorption, a low albumin level or a reduced bilirubin-albumin binding capacity and/or trouble establishing breastfeeding; such events favor increased bilirubin levels. ${ }^{2}$

In addition to what has been described, NBIs have a vulnerable CNS. In neonates, the bloodbrain barrier is unstable, especially in pathological perinatal circumstances that increase its capillary permeability: perinatal asphyxia, acidosis, and sepsis. ${ }^{16}$

In the CNS, bilirubin causes toxic effects, oxidative damage, and irreversible injuries. Consequences include a transient (bilirubininduced neurologic dysfunction) or chronic and devastating impact (chronic bilirubin encephalopathy), central hearing loss, and even death. In PNBIs, these conditions may occur with low bilirubin levels, cause subtle neurological disabilities, and lead to manifestations that vary even among patients born at the same gestational age. ${ }^{17,18}$

\section{Bilirubin binding to albumin}

Albumin is the most common serum protein. It accounts for $50-60 \%$ of total plasma protein in human beings. It binds to a wide variety of endogenous and exogenous ligands, which it carries. ${ }^{19}$ Albumin-bilirubin binding is the main protective factor of cells; it depends on their levels and on albumin affinity for bilirubin. ${ }^{19,20}$

Only FIB, which is not bound to albumin, crosses the blood-brain barrier, passes through the membranes, and enters cells. ${ }^{20}$ FIB increases as IB reaches its total albumin binding capacity ( $1 \mathrm{~g}$ of human serum albumin binds to $8 \mathrm{mg}$ of IB in term NBIs, but this is lower in PNBIs).

Binding is reversible. The binding-unbinding balance is dynamic and varies depending on $\mathrm{pH}$ and temperature. ${ }^{19}$ Conditions like hypothermia, hypoxia, acidosis, and asphyxia favor neurotoxicity by breaking the binding and generating FIB. ${ }^{21,22}$ Certain drugs like ceftriaxone and ibuprofen compete with bilirubin for albumin binding. ${ }^{19} \mathrm{~A}$ lipid intake of more than $1.5 \mathrm{~g} / \mathrm{kg}$ significantly reduces the bilirubin-albumin binding capacity and increases FIB levels in PNBIs born at less than 28 weeks of gestational age. ${ }^{21}$ In these patients, the bilirubin-albumin ratio would be a better predictor of bilirubin-induced neurotoxicity than TSB levels, although further studies are required. ${ }^{19,21}$

\section{Cell toxicity by bilirubin}

Bilirubin-induced neurotoxicity involves complex cascades of molecular and cellular events. It alters the blood-brain barrier and affects some CNS regions particularly. It has multiple effects on neurons and glial cells, cellular and mitochondrial membranes, and the endoplasmic reticulum. It causes a failure in mitochondrial energy production and excitotoxicity due to glutamate receptor stimulation, increased intracellular calcium levels, necrosis, and apoptosis. It also triggers inflammatory factor and oxidative stress cascades, which may cause abnormalities and even interrupt the cell cycle ${ }^{2}$ (Figure 2).

\section{Bilirubin and cell membranes}

Bilirubin is amphiphilic, which implies two 
properties. One property is that its ends have different characteristics (a polar or hydrophilic end and a nonpolar or hydrophobic end). The other property is that it is long enough so that each end expresses its own solubility characteristics. ${ }^{23}$

Several studies have suggested that cell membranes are the initial site in the bilirubininduced toxicity mechanism. The hydrophobic end of bilirubin shows a high affinity for membrane lipids. ${ }^{2,24,25}$ Zucker et al. demonstrated that, given the amphiphilic nature of bilirubin, interaction with phospholipid bilayers takes place in the outer polar region of the cell, close to the membranewater interface. ${ }^{24}$ After binding to the membrane, a molecular phospholipid alteration, membranebound ATPase inhibition, and lipid peroxidation occur, among other adverse events. ${ }^{25}$

In mitochondrial membranes, bilirubin causes analogous changes, with an interruption in energy production. ${ }^{26}$ The inhibition of several enzymatic systems and ribonucleic acid (RNA), protein, and carbohydrates synthesis in the CNS contribute to cell damage. ${ }^{20}$ Further studies are required to investigate the characteristics of bilirubin binding to membranes, its exact location, and the therapeutic possibilities that may be established with such knowledge. ${ }^{2}$

\section{Neuroinflammation}

Conditions associated with severe systemic inflammation, like sepsis, necrotizing enterocolitis, and fetal inflammatory response syndrome (chorioamnionitis with funisitis), favor bilirubin-induced neurotoxicity. ${ }^{2}$ These effects are greater in the immature cells of PNBIs. ${ }^{27,28}$ The proinflammatory cell environment generated by bilirubin activates microglia and releases tumor necrosis factor alpha (TNF- $\alpha$ ), interleukin $1 b$, and interleukin 6, which promote neurotoxicity ${ }^{18,29}$ (Figure 2).

\section{Blood-brain barrier}

The blood-brain barrier, a complex and dynamic interface between the CNS and blood, maintains the CNS homeostasis. Its integrity is warranted by the characteristics of binding and adherence of the endothelial cells that make it up.

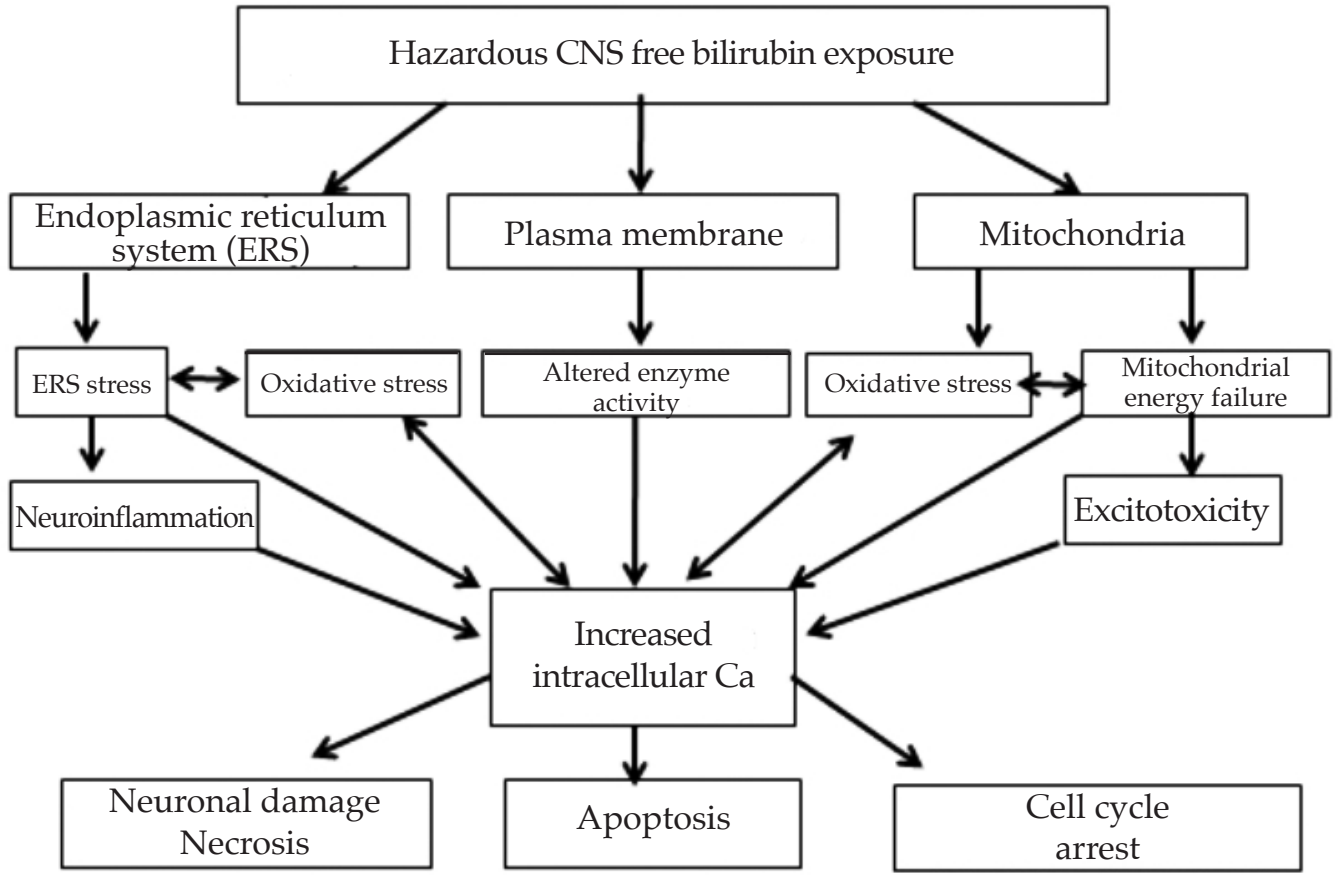

CNS: central nervous system; ERS: endoplasmic reticulum system; Ca: calcium.

Group of bilirubin toxicity mechanisms: neuronal damage, necrosis, and programmed cell death, all instances at which injury becomes irreversible. From Watchko J. Bilirubin-Induced Neurotoxicity in the Preterm Neonate. Clin Perinatol 2016;43:297-311. 
Palmela et al., demonstrated that the effect of bilirubin on the blood-brain barrier depended on exposure time. In this study, a 4-hour exposure increased, at the level of endothelial cells, vascular endothelial growth factor and caveolin levels. The latter are proteins contained in small plasma membrane invaginations called caveolae and modulate permeability, signal transduction, and endocytosis. They are important in blood-brain barrier alterations and may be key for future therapeutic findings.

With prolonged exposure ( 24 hours), cell binding and adherence vanished and permeability increased. A 72-hour exposure triggered autophagy and apoptosis responses. These results allow to infer the importance of establishing an early treatment, even in the presence of subtle neurological damage, before the alteration of the blood-brain barrier permeability and the initiation of apoptosis in the endothelial and CNS cells, with severe and irreversible neurological manifestations. ${ }^{30}$

\section{Oxidative stress}

The imbalance between the production of free radicals (FRs) and the antioxidant defense capacity causes oxidative stress, to which PNBIs are especially vulnerable. Low to moderately high bilirubin levels account for a potent antioxidant. ${ }^{8-11}$ However, paradoxically, once certain threshold is exceeded, it is harmful for the developing brain. Different studies have demonstrated that oxidative stress is a key mechanism in bilirubininduced neurotoxicity. 6,29

Vodret et al., referred that the cerebellum was the most affected organ by oxidative stress and inflammation due to high bilirubin levels. ${ }^{5}$ Rawat et al., conducted a study that found chromosomal translocations and deletions that caused oxidative damage in deoxyribonucleic acid (DNA) and an interruption of the cell function cycle that increased with bilirubin exposure time. ${ }^{7}$ Basu et al., described such damage even in the case of low bilirubin levels ${ }^{8}$ (Figure 2).

\section{Antioxidant effect}

The best-known biological effects of bilirubin are its harmful effects on the CNS of NBIs when it reaches high serum levels. However, molecular biology studies in adults have evidenced that bilirubin is a cytoprotective antioxidant. ${ }^{8-11}$

FRs originate in the mitochondrial respiratory chain and are responsible for cellular oxidative damage. ${ }^{31}$ In aerobic organisms, cellular respiration originates ATP and water molecules. In oxygen deprivation situations, this metabolic function generates FRs (superoxide anion, hydrogen peroxide, hydroxyl radical), which cause the lipid peroxidation of cell membranes and are involved in cancer pathophysiology, aging, heart disease, chronic inflammation, and infections. ${ }^{32}$ In PNBIs, FRs participate in disease pathogenesis, such as bronchopulmonary dysplasia, periventricular leukomalacia, necrotizing enterocolitis, patent ductus arteriosus, and retinopathy of prematurity. ${ }^{33}$

Birth is an event that causes oxidation due to the change in oxygen pressures. PNBIs also experience a high tissue growth rate and immature or absent antioxidant molecules, such as superoxide dismutase, catalase, vitamins $\mathrm{E}$ and $\mathrm{C}$, and beta-carotenes. ${ }^{34}$

In 1937, Najib-Farah was the first person to postulate that bilirubin was part of a protective mechanism designed to overcome infections. ${ }^{35}$ In 1989, Glazer presented a hypothesis about the role of end products of metabolic pathways as cell protection agents (uric acid, the end product of purine metabolism, and taurine, the end product of cysteine metabolism). ${ }^{36}$ In this context, Stocker observed, in an in vitro study, that the antioxidant activity of bilirubin increased as oxygen levels decreased from $21 \%$ (environmental concentration) to $2 \%$ (physiologically relevant concentration, corresponding to that of tissues). Stocker documented that bilirubin had a greater action than alpha-tocopherol, the most effective lipid-soluble antioxidant in the human species. Stocker proposed the beneficial role of bilirubin as a product of metabolic waste, disruptor of the oxidation biological chain. ${ }^{37}$

The bilirubin metabolism level involved in antioxidant functions is that where $\mathrm{HO}$ and BVR enzymes act (Figure 1). HO, which is induced by heme and proinflammatory lesions, has antiapoptotic, antioxidant, and anti-inflammatory effects. It transforms the heme molecule into biliverdin. The metabolic pathway is completed once biliverdin is reduced by BVR to bilirubin. ${ }^{38,39}$

New hypotheses have suggested that, based on the extent of oxidation to which cells are exposed and in order to maintain their antioxidant homeostasis, they have bilirubin recovery pathways. These include the biliverdinbilirubin redox cycle in the endoplasmic reticulum system and the cytochrome $\mathrm{P} 450$ pathway in the mitochondria, both with a reverse action compared to bilirubin metabolism ${ }^{10,38}$ (Figure 1). 
In these metabolic pathways, bilirubin is oxidized to biliverdin, which will be once again reduced to bilirubin through the BVR enzyme. Based on such redox sequences, bilirubin captures FRs, detoxifies up to 10000 times FR excess, and protects membrane lipids from peroxidation. ${ }^{40}$

In amphibians, reptiles, and birds, heme metabolism stops at the level of biliverdin. In mammals, the antioxidant power of bilirubin justifies that the heme metabolic pathway progresses to bilirubin formation, although it requires a greater amount of enzymatic agents, a greater energy consumption, and albumin binding so it is transported and conjugated for excretion..$^{38}$

In adults, several publications established a relation between the antioxidant property of bilirubin and a better prognosis in diseases that cause oxidative stress: Alzheimer's disease, ischemia-reperfusion injuries, cardiovascular disease, diabetes, inflammation, cancer. In Gilbert's syndrome, which causes high IB levels, the frequency of ischemic heart disease is lower than in the general population (2\% versus $12 \%){ }^{38}$

Science has made advances in the study of cellular damage caused by oxidative stress. Accurately establishing the role of FRs and molecules that are part of antioxidant defenses is a major challenge. In this context, a better understanding of bilirubin's antioxidant capacity, its intermediate metabolites, and its complex enzymatic structure, will probably allow to take advantage of its potential benefit for PNBIs.

\section{Excitotoxicity}

According to in vivo and in vitro studies, excitotoxicity would be another mechanism through which bilirubin may harm the CNS. Glutamate, the main excitatory neurotransmitter of the CNS, and its receptor N-methyl-D-aspartate (NMDA) are involved. ${ }^{41}$

Glutamate alone is necessary, but not enough. Excitotoxicity would be preceded by a sustained failure in the production of mitochondrial energy, which would promote neuronal depolarization, and then a passive opening of the NMDA glutamate channels., ${ }^{2,41}$ Once such channels are open, the events typical of excitotoxicity are manifested:

a. Increased intracellular sodium and chloride levels, which cause cellular inflammation.

b. Calcium entry to the cell and calcium release from intracellular stores.

c. Activation of calcium-dependent enzymes that activate apoptosis and / or necrosis. ${ }^{42}$

Mitochondrial energy failure and excitotoxicity would explain the regional nature of bilirubin-induced CNS damage. ${ }^{2,41}$ In NBIs, the characteristics of globus pallidus neurons make them susceptible to all the events described here: a high baseline neuronal activity makes them susceptible to acute failures in mitochondrial energy and the high density of neuronal glutamate receptors may intensify excitotoxicity ${ }^{43}$ (Figure 2).

\section{CONCLUSIONS}

The characteristics of NBIs make them vulnerable to hyperbilirubinemia. It is a clinically harmful condition in these patients, so it is imperative to treat it in a timely and effective manner.

Bilirubin is a molecule with a dual behavior. It is a potent antioxidant that is present at a time of great exposure to oxidative damage: birth, especially in the case of PNBIs. However, in certain conditions, it is capable of causing severe and irreversible cell damage, which can be prevented with an adequate management. We still need to determine the exact FIB levels or the environmental conditions in which it acts to cause one or other effect.

In order to perfect treatment indications, it is necessary to overcome several challenges: establishing methods to measure FIB levels, detecting minimum bilirubin-induced neurologic dysfunction in an early manner, and deepening knowledge about its potential antioxidant benefits so as to weigh them against the risks for cell damage that may be also caused by bilirubin.

Once such scientific advances are made, phototherapy indications will probably need to be adapted to them for a greater accuracy in relation to treatment indications. In the meantime, treatment decisions should be customized to each patient and reflect expert consensus recommendations.

\section{REFERENCES}

1. Olusanya B, Kaplan M, Hansen T. Neonatal hyperbilirubinaemia: a global perspective. Lancet Child Adolesc Health. 2018; 2(8):610-20.

2. Watchko J. Bilirubin-induced neurotoxicity in the preterm neonate. Clin Perinatol. 2016; 43(2):297-311.

3. Riordan S, Shapiro S. Review of bilirubin neurotoxicity I: molecular biology and neuropathology of disease. Pediatr Res. 2020; 87(2):327-31.

4. Shapiro S, Riordan S. Review of bilirubin neurotoxicity II: preventing and treating acute bilirubin encephalopathy and kernicterus spectrum disorders. Pediatr Res. 2020; $87(2): 332-7$. 
5. Vodret S, Bortolussi G, Ja $\square$ prová J, Vitek L, et al. Inflammatory signature of cerebellar neurodegeneration during neonatal hyperbilirubinemia in Ugt1-/- mouse model. J Neuroin flammation. 2017; 14(1):64.

6. Bortolussi G, Codarin E, Antoniali G, Vascotto C, et al. Impairment of enzymatic antioxidant defenses is associated with bilirubin-induced neuronal cell death in the cerebellum of Ugt1 KO mice. Cell Death Dis. 2015; 6(5):e1739.

7. Rawat V, Bortolussi G, Gazzin S, Tiribelli C, et al. Bilirubininduced oxidative stress leads to DNA damage in the cerebellum of hyperbilirubinemic neonatal mice and activates DNA double-strand break repair pathways in human cells. Oxid Med Cell Longev. 2018; 2018:1801243.

8. Basu S, DeD, Dev Khanna H, Kumar A. Lipid peroxidation, DNA damage and total antioxidant status in neonatal hyperbilirubinemia. J Perinatol. 2014; 34(7):519-23.

9. Zahir F, Rabbani G, Khan R, Rizvi S, et al. The pharmacological features of bilirubin: The question of the century. Cell Mol Biol Lett. 2015; 20(3):418-47.

10. Gazzin S, Vitek L, Watchko J, Shapiro S, et al. A novel perspective on the biology of bilirubin in health and disease. Trends Mol Med. 2016; 22(9):758-68.

11. Stocker R. Turning the catabolism in usefulness-A jaundiced view. Clin Chem. 2011; 57(11):1612-3.

12. Torres-Cuevas I, Parra-Llorca A, Sánchez-Illana A, NuñezRamiro A, etal. Oxygen and oxidative stress in the perinatal period. Redox Biol. 2017; 12:674-81.

13. American Academy of Pediatrics Subcommittee on Hyperbilirubinemia. Management of hyperbilirubinemia in the newborn infant 35 or more weeks of gestation. Pediatrics. 2004; 114(1):297-316.

14. Fujiwara R, Haag M, Schaeffeler E, Nies A, et al. Systemic regulation of bilirubin homeostasis: potential benefits of hyperbilirubinemia. Hepatology. 2018; 67(4):1609-19.

15. Iwatani S, Nakamura H, Kurokawa D, Yamana K, et al. Fluorescent protein-based detection of unconjugated bilirubin in newborn serum. Sci Rep. 2016; 6:28489.

16. WisnowskiJL, Panigrahy A, Painter M, WatchkoJ. Magnetic resonance imaging of bilirubin encephalopathy: current limitations and future promise. Semin Perinatol. 2014; 38(7):422-8.

17. Shapiro SM. Kernicterus. In: Stevenson DK, Maisels MJ, Watchko JF (eds.). Care of the jaundiced neonate. New York: McGraw Hill; 2012.Pp.229-42.

18. Watchko JF, Maisels MJ. The enigma of low bilirubin kernicterus in premature infants: why does it still occur, and is it preventable? Semin Perinatol. 2014; 38(7):397-406. 19.

19. Amin S. Bilirubin binding capacity in the preterm neonate. Clin Perinatol. 2016; 43(2):241-57.

20. Hansen T. Biology of bilirubin photoisomers. Clin Perinatol. 2016; 43(2):277-90.

21. Amin SB. Clinical assessment of bilirubin-induced neurotoxicity in premature infants. Semin Perinatol. 2004; 28(5):340-7.

22. Amin SB, Harte T, Scholer L, Wang H. Intravenous lipid and bilirubin-albumin binding variables in premature infants. Pediatrics. 2009; 124(1):211-7.
23. Cox C, Gottlieb P. Amphipathic molecules modulate PIEZO1 activity. Biochem Soc Trans. 2019; 47(6):1833-42.

24. Zucker SD, Goessling W, Bootle EJ, Sterritt C. Localization of bilirubin in phospholipid bilayers by parallax analysis of fluorescence quenching. J Lipid Res. 2001; 42(9):1377-88.

25. Brito M, Brites D, Butterfield D. A link between hyperbilirubinemia, oxidative stress and injury to neocortical synaptosomes. Brain Res. 2004; 1026(1):33-43.

26. Rodrigues C, SoláS, Castro R, Laires P, et al. Perturbation of membrane dynamics in nerve cells as an early event during bilirubin-induced apoptosis. J Lipid Res. 2002; 43(6):885-94.

27. Brites D. The evolving landscape of neurotoxicity by unconjugated bilirubin: role of glial cells and inflammation. Front Pharmacol. 2012; 3:88.

28. Kitamura M. Control of NF-kB and inflammation by the unfolded protein response. Int Rev Immunol. 2011; 30(1):415.

29. Watchko J, Tiribelli C. Bilirubin-induced neurologic damage-mechanisms and management approaches. $N$ Engl J Med. 2013; 369(21):2012-30.

30. Palmela I, Sasaki H, Cardoso F, Moutinho M, et al. Timedependent dual effects of high levels of unconjugated bilirubin on the human blood-brain barrier lining. Front Cell Neurosci. 2012; 6:22.

31. Morita T. Heme oxygenase and atherosclerosis. Arterioscler Thromb Vasc Biol. 2005; 25(9):1786-95.

32. Kapitulnik J. Bilirubin: an endogenous product of heme degradation with both cytotoxic and cytoprotective properties. Mol Pharmacol. 2004; 66(4):773-9.

33. Ozsurekci Y, Aykac K. Oxidative stress related diseases in newborns. Oxid Med Cell Longev. 2016; 2016:2768365.

34. Stocker R, Yamamoto Y, McDonagh A, Glazer A, et al. Bilirubin is an antioxidant of possible physiological importance. Science. 1987; 235(4792):1043-6.

35. Farah N. Defensive role of bilirubinaemia in pneumococcal infection. Lancet. 1937; 229(5922):505-6.

36. Glazer AN. Light guides. Directional energy transfer in a photosynthetic antenna. J Biol Chem. 1989; 264(1):1-4.

37. Stocker R, Perrella MA. Heme oxygenase-1. A novel drug target for atherosclerotic diseases? Circulation. 2006; 114(20):2178-89.

38. Otero Regino W, Velasco H, Sandoval H. Papel protector de la bilirrubina en el ser humano. Rev Col Gastroenterol. 2009; 24(3):293-301.

39. Ryter SW, Alam J, Choi AM. Heme oxigenase-1/carbon monoxide: from basic science to therapeutic applications. Physiol Rev. 2006; 86(2):583-650.

40. Sedlack TW, Snyder SH. Bilirubin benefits: cellular protection by a biliverdin reductase antioxidant cycle. Pediatrics. 2004; 113(6):1776-82.

41. Johnston M. Excitotoxicity in perinatal brain injury. Brain Pathol. 2005; 15(3):234-40.

42. Slemmer JE, De Zeeuw CI, Weber JT. Don't get too excited: mechanisms of glutamate-mediated Purkinje cell death. Prog Brain Res. 2005; 148:367-90.

43. Greenamyre T, Penney JB, Young AB, Hudson C, et al. Evidence for transient perinatal glutaminergicinnervation of globus pallidus. J Neurosci. 1987; 7(4):1022-30. 